\title{
Article
}

\section{Imprints of radial migration on the Milky Way's metallicity distribution functions}

Loebman, Sarah R., Debattista, Victor P, Nidever, David L., Hayden, Michael R., Holtzman, Jon A., Clarke, Adam J., Roškar, Rok and Valluri, Monica

Available at https://clok.uclan.ac.uk/13851/

Loebman, Sarah R., Debattista, Victor P orcid iconORCID: 0000-0001-79020116, Nidever, David L., Hayden, Michael R., Holtzman, Jon A., Clarke, Adam J., Roškar, Rok and Valluri, Monica (2016) Imprints of radial migration on the Milky Way's metallicity distribution functions. The Astrophysical Journal Letters, 818 (1). L6. ISSN 2041-8213

It is advisable to refer to the publisher's version if you intend to cite from the work. http://dx.doi.org/10.3847/2041-8205/818/1/L6

For more information about UCLan's research in this area go to http://www.uclan.ac.uk/researchgroups/ and search for <name of research Group>.

For information about Research generally at UCLan please go to http://www.uclan.ac.uk/research/

All outputs in CLoK are protected by Intellectual Property Rights law, including Copyright law. Copyright, IPR and Moral Rights for the works on this site are retained by the individual authors and/or other copyright owners. Terms and conditions for use of this material are defined in the policies page. 


\title{
IMPRINTS OF RADIAL MIGRATION ON THE MILKY WAY'S METALLICITY DISTRIBUTION FUNCTIONS
}

\author{
Sarah R. Loebman ${ }^{1,8}$, Victor P. Debattista ${ }^{2}$, David L. Nidever ${ }^{1,3,4}$, Michael R. Hayden $^{5}$, Jon A. Holtzman ${ }^{6}$, \\ AdAM J. Clarke ${ }^{2}$, ROK RošKar ${ }^{7}$, AND MONICA VAlluri ${ }^{1}$ \\ ${ }^{1}$ Department of Astronomy, University of Michigan, 1085 S. University Avenue, Ann Arbor, MI48109-1107, USA; sloebman@umich.edu \\ 2 Jeremiah Horrocks Institute, University of Central Lancashire, Preston PR1 2HE, UK; vpdebattista@uclan.ac.uk \\ ${ }^{3}$ Steward Observatory, 933 North Cherry Avenue, Tucson, AZ 85719, USA \\ ${ }^{4}$ Large Synoptic Survey Telescope, 950 North Cherry Avenue, Tucson, AZ 85719, USA; dnidever@1sst.org \\ ${ }^{5}$ Laboratoire Lagrange, Université Côte d'Azur, Observatoire de la Côte d'Azur, CNRS, Blvd de l'Observatoire, CS 34229, F-06304 Nice cedex 4, France; \\ mhayden@oca.eu \\ ${ }^{6}$ New Mexico State University, Las Cruces, NM 88003, USA \\ ${ }^{7}$ Research Informatics, Scientific IT Services, ETH Zürich, Weinbergstrasse 11, CH-8092 Zürich, Switzerland \\ Received 2015 November 16; accepted 2016 January 8; published 2016 February 3
}

\begin{abstract}
Recent analysis of the SDSS-III/Apache Point Observatory Galactic Evolution Experiment (APOGEE) Data Release 12 stellar catalog has revealed that the Milky Way's (MW) metallicity distribution function (MDF) changes shape as a function of radius, transitioning from being negatively skewed at small Galactocentric radii to positively skewed at large Galactocentric radii. Using a high-resolution, $N$-body $+\mathrm{SPH}$ simulation, we show that the changing skewness arises from radial migration-metal-rich stars form in the inner disk and subsequently migrate to the metal-poorer outer disk. These migrated stars represent a large fraction $(>50 \%)$ of the stars in the outer disk; they populate the high-metallicity tail of the MDFs and are, in general, more metal-rich than the surrounding outer disk gas. The simulation also reproduces another surprising APOGEE result: the spatially invariant high- $[\alpha / \mathrm{Fe}]$ MDFs. This arises in the simulation from the migration of a population formed within a narrow range of radii $(3.2 \pm 1.2 \mathrm{kpc})$ and time $(8.8 \pm 0.6 \mathrm{Gyr}$ ago), rather than from spatially extended star formation in a homogeneous medium at early times. These results point toward the crucial role radial migration has played in shaping our MW.
\end{abstract}

Key words: Galaxy: abundances - Galaxy: disk - Galaxy: evolution - Galaxy: formation - Galaxy: kinematics and dynamics - Galaxy: structure

\section{INTRODUCTION}

Observationally, it is challenging to explore the chemical evolution of the Milky Way (MW) for several reasons. First, stars of a given type span a wide range of distances and are therefore "smeared out" in apparent magnitude space. Second, high-resolution optical spectroscopy is only possible for relatively bright stars. Finally, the majority of stars in the Galactic disk are obscured by dust at visual wavelengths. Given these obstacles plus the finite range of Hipparcos parallaxes, most past chemical and/or kinematic studies have focused on the solar neighborhood (e.g., Steinmetz et al. 2006; Holmberg et al. 2009; Fuhrmann 2011; Ramírez et al. 2013) or high latitudes (e.g., Steinmetz et al. 2006; Yanny et al. 2009) where the dust extinction is low. This approach has allowed for the development of useful insight into our local environment and the stellar halo, but it has given only a partial view of the Galactic disk.

Recently, SDSS-III's Apache Point Observatory Galactic Evolution Experiment (APOGEE; Eisenstein et al. 2011) set out to characterize the MW disk using a different approach. APOGEE is a spectroscopic survey that pushes through the dust "barrier" by operating in the near-infrared. In 2015, APOGEE released its third year catalog (DR12; Alam et al. 2015; Holtzman et al. 2015) containing 140,000 stars, dominated by red giant branch (RGB) stars that probe to large distances $(\sim 10 \mathrm{kpc})$.

These data have already yielded important insights into the structure and chemistry of the disk, including the generation of

\footnotetext{
${ }^{8}$ Michigan Society of Fellows
}

mean metallicity maps over a large radial range $(R<15 \mathrm{kpc}$, where $R$ is Galactocentric radius; Hayden et al. 2014), the quantification of the radial metallicity gradient outside $R \sim 5 \mathrm{kpc}$ (Hayden et al. 2014), and the confirmation of a prominent bimodality in $[\alpha / \mathrm{Fe}]$ for stars with low to intermediate metallicities (Anders et al. 2014; Nidever et al. 2014). Additionally, using the red clump catalog (Bovy et al. 2014), Nidever et al. (2014) found that the chemical abundance pattern of high- $[\alpha / \mathrm{Fe}]$ stars is remarkably uniform throughout $5<R<11 \mathrm{kpc}$. Nidever et al. (2014) interpreted this as indicating that the early evolution of the stellar disk was characterized by a similar star formation history from a a wellmixed interstellar medium (ISM). Their simple Galactic chemical evolution model for the high- $[\alpha / \mathrm{Fe}]$ sequence also implied a short gas consumption timescale (i.e., very active star formation early on).

More recently, Hayden et al. (2015, hereafter H15) used $\sim 70,000$ RGB stars (spanning $3<R<15 \mathrm{kpc}$ ) from DR12 to analyze the metallicity distribution function (MDF) in the midplane and found that the shape of the MDF transitions from being negatively skewed in the inner galaxy to positively skewed in the outer Galaxy, with a nearly symmetric MDF in the solar neighborhood. H15 found that heating and epicyclic motion do not alter the shape of the MDF enough to explain the APOGEE observations; rather, heating broadens but does not alter the skewness of the MDFs. H15 conclude that the positive skewness in the outer Galaxy is most plausibly explained by the movement of more metal-rich stars from the inner Galaxy outward via radial migration. 
Radial migration due to transient spirals was first described by Sellwood \& Binney (2002). In this process, energy and angular momentum changes occur from interactions with transient spiral arms, which move stars at the corotation resonance inward or outward in radius while preserving their nearly circular orbits. Stars migrated by this process do not experience significant heating because of it (Roškar et al. 2013), while stars migrated by other processes do (Minchev \& Famaey 2010). Several groups have found observational evidence that the solar neighborhood has been contaminated by transient spiral migration (e.g., Liu \& van de Ven 2012; Yu et al. 2012). In this Letter, we consider a simulation in which radial migration is primarily due to the corotation resonance mechanism of Sellwood \& Binney (2002) and where the heating of stars is modest, consistent with what is seen in the MW. Radial migration has been studied both analytically (e.g., Schönrich \& Binney 2009; Daniel \& Wyse 2015) and in numerical simulations (e.g., Roškar et al. 2012; Solway et al. 2012; Vera-Ciro et al. 2014; Grand et al. 2015). Migration in the simulation we consider here is dominated by spirals, making it an ideal testbed for comparison with the APOGEE data. Roškar et al. (2008b) previously demonstrated that this simulation reproduces the MW's MDF in the solar cylinder; here, we extend this analysis to the full APOGEE footprint.

In what follows, we revisit two of the recent APOGEE results: the changing shape of the Galactic MDF in the midplane and the constancy of the high- $[\alpha / \mathrm{Fe}] \mathrm{MDF}$ in all spatial volumes. Using a numerical simulation, we provide strong support for H15's interpretation of the origin of the change in the MDF skewness and provide a novel explanation for the homogeneity of the high- $[\alpha / \mathrm{Fe}]$ sequence. That is, we show that both results are a natural consequence of a single persistent secular process: radial migration, of an early-forming population generated in a limited volume in the case of the $[\alpha / \mathrm{Fe}]-$ rich population. From this, we conclude that radial migration likely significantly influenced the MW throughout its history.

\section{SIMULATION}

In this Letter, we analyze the same simulation that was used in Loebman et al. (2011). This simulation is a re-run of the fiducial case discussed in Roškar et al. (2008a, 2008b); it includes metal diffusion, but is otherwise identical to the earlier model.

A detailed description of the simulation's implementation can be found in Roškar et al. (2012). Briefly, the simulation is evolved in isolation using the $N$-body + smooth particle hydrodynamics code GASOLINE (Wadsley et al. 2004). This allows for a high mass and force resolution (baryonic softening of $50 \mathrm{pc}$ ). The initial conditions include two NFW (Navarro et al. 1997) halos each containing $10^{6}$ particles, one of dark matter with a mass of $10^{12} M_{\odot}$ and the other of gas in hydrostatic equilibrium with a mass of $10^{11} M_{\odot}$. The gas is set with an initial rotation with $\lambda=0.065 .^{9}$ As the simulation progresses, the gas cools and collapses into a disk that forms stars according to a standard prescription: star particles form from gas with a temperature $<1.5 \times 10^{4} \mathrm{~K}$ and a density $>0.1$ $\mathrm{amu} / \mathrm{cc}$ (Stinson et al. 2006).

\footnotetext{
9 In earlier works, we reported $\lambda=0.039$. Here, we have recalculated $\lambda$ for the gas only according to Equation (1) in Debattista et al. (2015).
}

GASOLINE generates metals according to a subgrid model of supernovae and asymptotic giant branch star enrichment (Stinson et al. 2006). This prescription uses yields from Raiteri et al. (1996; supernovae II) and Thielemann et al. (1986; supernovae Ia) and includes the effects of stellar winds on mass redistribution (Weidemann 1987). Diffusion of metals through the ISM follows a subgrid model of eddy turbulence based on the local smoothing length and velocity gradients (Smagorinsky 1963; Wadsley et al. 2008).

As in Loebman et al. (2011), we define high- $[\alpha / \mathrm{Fe}]$ stars in the simulation as star particles with $[\mathrm{O} / \mathrm{Fe}] \gtrsim-0.05$. This threshold is set lower than the APOGEE threshold $([\alpha / \mathrm{Fe}] \gtrsim$ 0.18 ) for two reasons: (1) unlike the nascent MW disk, the simulation starts with unenriched gas, and (2) there is uncertainty in the adopted yields. These discrepancies result in a relative offset at the end of the simulation of $\sim 0.2 \mathrm{dex}$, but as we showed in Loebman et al. (2011) and Roškar et al. (2013), the global trends in enrichment match expectations for the MW. Therefore, throughout this work, we will focus on the relative trends in the MDFs, which are much more robust, rather than absolute comparisons.

At the end of the simulation, the galaxy contains more than $2 \times 10^{6}$ star particles. The final disk scale length is $2.5 \mathrm{kpc}$, and the disk has a break radius of $10 \mathrm{kpc}$. As done in Roškar et al. (2013) and Loebman et al. (2011), stars that have moved radially by more than $2 \mathrm{kpc}$ from their birth location are classified as radial migrators, while stars that have moved less than $2 \mathrm{kpc}$ are classified as in situ stars. While $25 \%$ of the stars in the entire simulation have radially migrated, the relative fraction of migrators varies with radius. Heating plays a relatively minor role in bringing migrators into the solar cylinder; at the end of the simulation, the majority of solar cylinder migrators are on nearly circular orbits. For instance, in the solar cylinder, $80 \%$ of the migrators and $92 \%$ of the in situ star particles have a circularity $x=J_{z} / J_{c}$ greater than 0.90 (corresponding to an average and maximum epicyclic radius of $1.4 \pm 0.2 \mathrm{kpc}$ and $1.9 \mathrm{kpc}$, respectively), while beyond the disk break $(R>10 \mathrm{kpc}), 75 \%$ of the migrators and $99 \%$ of the in situ star particles have a circularity above 0.90 .

\section{RESULTS}

\subsection{MDF of All Stars}

As noted in the previous section, stars in this model undergo significant radial migration; for example, $55 \%$ of stars ending in the solar cylinder $(7.5 \leqslant R / \mathrm{kpc} \leqslant 8.5)$ have moved in excess of $2 \mathrm{kpc}$ from their birth radii (Roškar et al. 2008b). Thus, the MDF of stars in a particular radial bin will evolve with time due to two effects: ongoing in situ star formation and the arrival of stars that formed elsewhere in the disk with a different chemical evolution history.

Figure 1 shows the MDF of stars as a function of their radius of formation. We can think of this as a control case: it is the MDF we would expect if there was no heating or radial migration within the simulation. As expected, the MDF of stars forming in the interior of the disk is peaked at higher metallicities than the MDF of stars that form in the exterior of the disk. Regardless of radial bin considered, the generic shape of the formation MDF is the same: it is peaked at relatively high metallicities and has a long tail toward lower metallicities. This shape, with skewness toward the low 


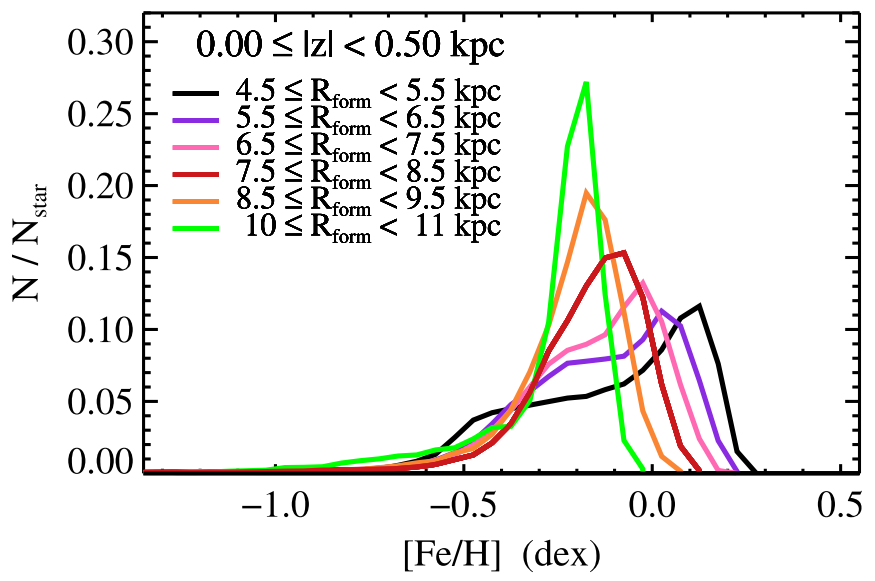

Figure 1. Metallicity distribution function (MDF) as a function of formation radius.

metallicities, is a generic prediction of simple chemical evolution models (B. Andrews et al. 2016, in preparation).

H15 found that the skewness of the APOGEE MDFs at large radii is not well described by this distribution. Instead, a long tail of high-metallicity stars is observed at large radii for stars selected in the plane of the disk (see H15; Figure 5, bottom panel). H15showed that if heating effects are included, the MDF is broadened, but the shape of the distribution, including the sense of its skewness, is maintained (see H15, Section 4.2 and Figure 8).

In Figure 2, we generate MDFs from stars at the end of the simulation after significant radial migration has occurred. Here, we see the same global trends as is seen in the APOGEE data. Particularly, in the plane of the disk, the shape of the distribution shifts from being skewed toward low metallicities at small radii to having no skewness in the solar cylinder, and finally being skewed toward high metallicities at large radii. Beyond $\sim 5 \mathrm{kpc}$, gas in each radial bin (Figure 2, bottom panel) is not metal-rich enough to have produced the high-metallicity stars. When volumes away from the plane of the disk are considered, the skewness toward high metallicities decreases in the same fashion as in the APOGEE data (see H15; Figure 5, middle and top panels).

For a more systematic comparison between the simulation and observations, we can quantify the skewness of each MDF with a single number. ${ }^{10}$ Figure 3 illustrates the skewness measured in each radial bin within the mid-plane $(0 \leqslant|z| / \mathrm{kpc}<0.5)$. The trend in the simulation shows remarkable agreement with the APOGEE results; in both cases, there is a steady increase from negative skewness to positive skewness with increasing radius with the turnover occurring at the solar radius. The right panel of Figure 3 illustrates the fraction of stars that are migrated in each radial bin in the mid-plane of the simulation. Note that the larger the radii considered, the larger the fraction of migrated stars and the larger the positive skewness. While it is tempting to read off the migrated fraction for APOGEE given a particular skewness, we

\footnotetext{
10 There is substantial literature on the optimal way to measure skewness (see, for instance, Doane \& Seward 2011). So that we are not unduly influenced by outliers, we opt to fit a skewed normal model (Azzalini \& Capitanio 2009) to each MDF and utilize the skewness parameter that is returned by the best-fit model. This method is less sensitive to outliers, provides an intuitive description of skewness, and generates a good global fit to the data. Our adopted metric yields a consistent trend as a function of radius as the results presented in $\mathrm{H} 15$.
}

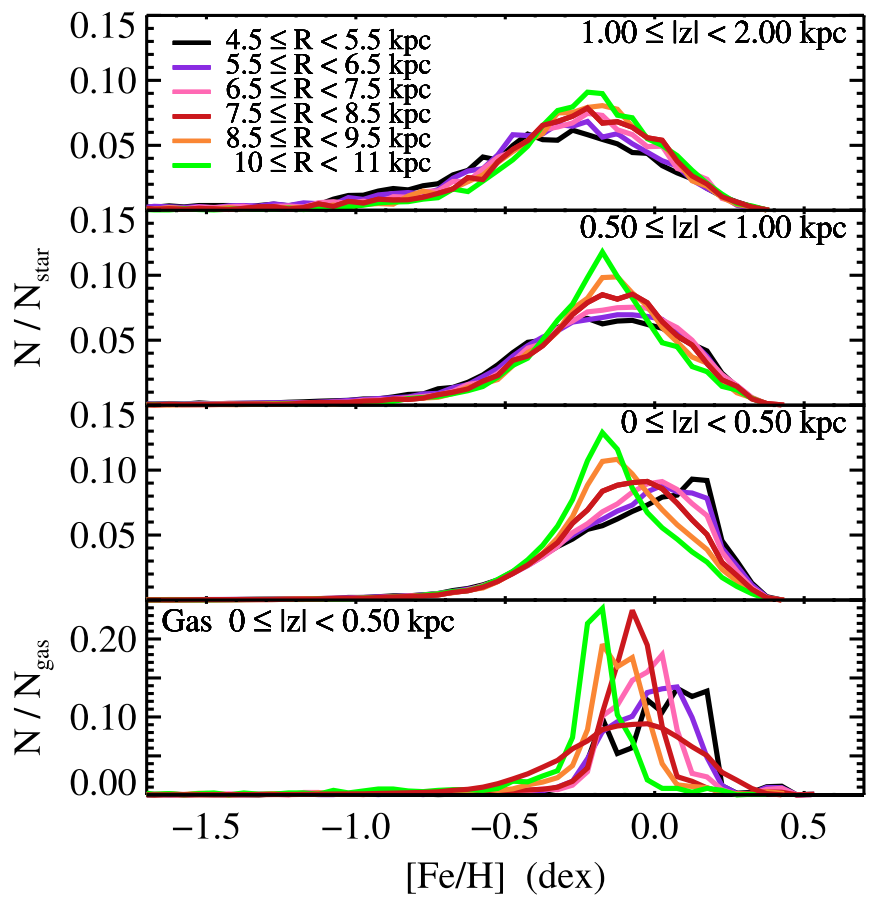

Figure 2. MDF as a function of present-day radius for all stars in the simulation. Varying distances from the mid-plane are considered: (top panel) $1.0 \leqslant|z| / \mathrm{kpc}<2.0$, (top middle panel) $0.5 \leqslant|z| / \mathrm{kpc}<1.0$, and (bottom middle panel) $0 \leqslant|z| / \mathrm{kpc}<0.5$. The gas MDF is also shown for comparison (bottom panel).

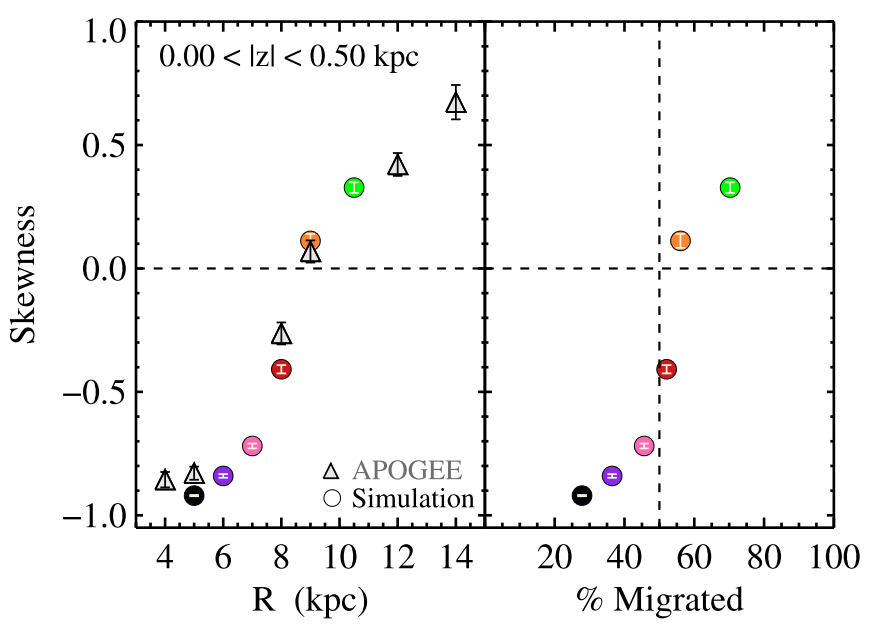

Figure 3. Left: the skewness of simulation (circles) and APOGEE (triangles) MDFs as a function of the present-day radius within the mid-plane $(0 \leqslant|z| / \mathrm{kpc}<0.5)$. Right: the trend in the simulation between skewness and the fraction of migrated stars; note that the color of the circles between the two panels is the same.

note that the maximum APOGEE skewness exceeds the maximum simulated skewness. Thus, only the trend should be considered, rather than the absolute migrated fraction, when drawing comparisons between the APOGEE skewness and the simulation.

In the insets in Figure 4, we show a direct comparison between the simulation and the APOGEE data; while a small offset is present, clearly the MDFs are well matched without the need of any tuning. Why is the simulation able to reproduce the observed shift in the shape of the MDF? In Figure 4, we look in detail at the in situ and migrated stars in the plane of the 


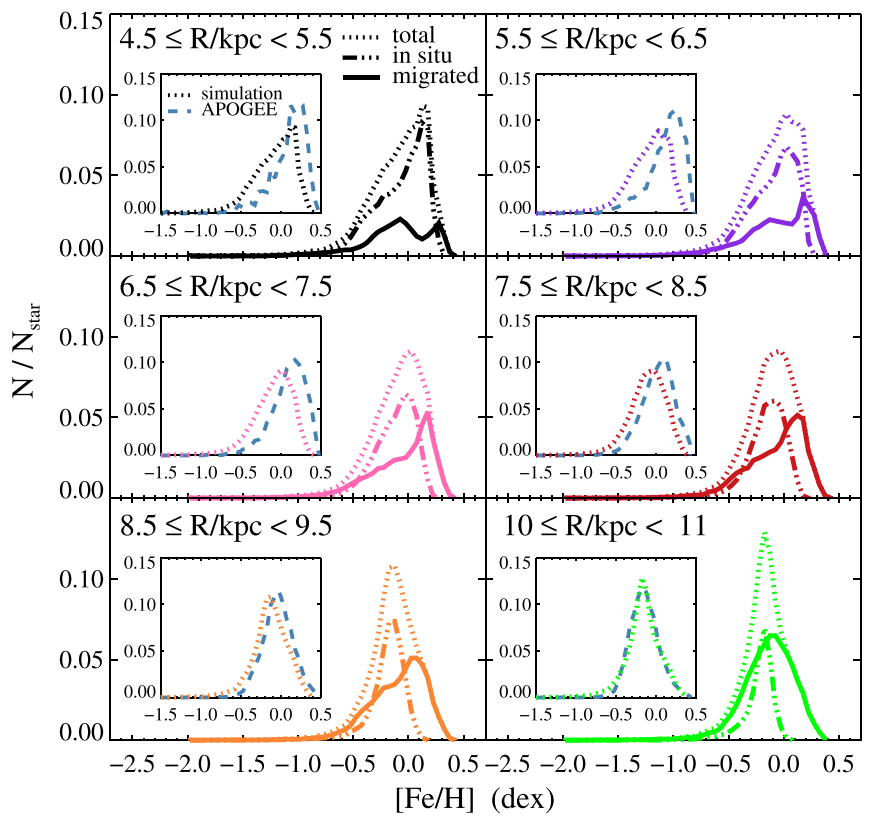

Figure 4. MDF for the total (dotted line), in situ (dashed-dotted line), and migrated (solid line) populations. All three MDFs within each panel have been normalized to the total number (in situ + migrated) of stars within the volume. That is, at each metallicity, the in situ and migrated values sum to the value shown in the total curve. Inset: total MDF for the simulation (dotted line) and APOGEE (dashed line) data.

disk. At all radial bins, the peak of the total MDF is set by the peak of the in situ population. However, beyond $\sim 5 \mathrm{kpc}$, the peak of the migrated population's MDF occurs at a metallicity where there are very few in situ stars-in these volumes, the gas is too metal-poor to produce them. Thus, the stars with the highest metallicities are necessarily migrated, and it is the offset between the peak of the in situ and the peak of the migrated population that generates the high-metallicity tail.

We see from Figure 3 that with increased radius comes an increased fraction of migrators. As the fraction of migrators rises, the MDF becomes more positively skewed. This is a result of the net outward flow of migrated stars. While it is equally likely for radial migration to move an individual star inward or outward in radius, given the exponential nature of the disk, the net movement of migrators is outward. Thus, MDFs in the outer regions of the galaxy are polluted by metal-rich stars that form in the interior of the disk. Over time, radial migration builds up the metal-rich tail of the MDF.

\section{2. $M D F$ of High- $[\alpha / F e]$ Stars}

Using red clump stars from APOGEE, Nidever et al. (2014) found that the high- $[\alpha / \mathrm{Fe}]$ chemical abundance patterns (in the $[\alpha / \mathrm{Fe}]$ versus $[\mathrm{Fe} / \mathrm{H}]$ plane) are the same (within $10 \%$ ) throughout the Galaxy. Nidever et al. (2014) interpreted the uniformity as an indication that the early Galaxy had a wellmixed ISM. That is, a well-mixed layer of gas was present throughout the MW at early times and experienced a high specific star formation rate throughout, forming stars with a similar chemistry everywhere.

$\mathrm{H} 15$ revisited the high- $[\alpha / \mathrm{Fe}]$ APOGEE stars and generated MDFs for this population (see Figure 6 in H15). Consistent with Nidever et al. (2014), H15 found remarkable uniformity in the high- $[\alpha / \mathrm{Fe}] \mathrm{MDFs}$. In the simulation, we also select high- $[\alpha / \mathrm{Fe}]$ stars by imposing a flat cut on $[\mathrm{O} / \mathrm{Fe}]$ (see Section 2 for details).

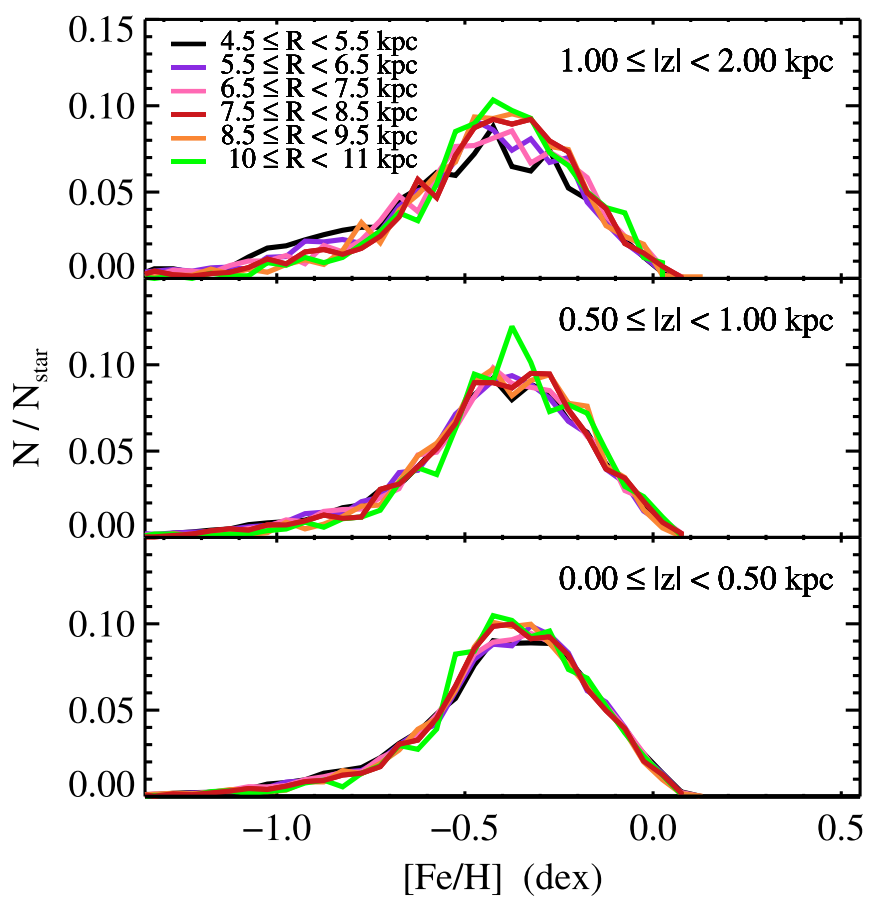

Figure 5. Same as Figure 2, but for the high- $[\alpha / \mathrm{Fe}]$ stars.

Figure 5 shows the resulting MDFs for this high- $[\alpha / \mathrm{Fe}]$ population. Without any fine-tuning, the simulation generates the same global fingerprint as seen in H15's high- $[\alpha / \mathrm{Fe}]$ population. Regardless of the radius considered, the high- $[\alpha / \mathrm{Fe}]$ MDF has a remarkably similar shape, peak, and width; a twosample Kolmogorov-Smirnov test between all the MDFs in the mid-plane finds that the average probability that these MDFs are drawn from a single distribution is $>90 \%$. Why is this the case?

In the simulation, the high- $[\alpha / \mathrm{Fe}]$ stars formed in one small region $(3.2 \pm 1.2 \mathrm{kpc})$ in a narrow timespan $(8.8 \pm 0.6 \mathrm{Gyr}$ ago $)$ in the inner Galaxy and then migrated out to their present location, all over the Galaxy. Because they were formed in a relatively small region of well-mixed gas, these stars are chemically alike; for this reason, regardless of the present-day radius considered, the high- $[\alpha / \mathrm{Fe}] \mathrm{MDF}$ looks identical. It is worth noting that Figure 5 shows a shallow $\left(-0.03 \mathrm{dex} \mathrm{kpc}^{-1}\right)$ negative vertical gradient in the peak metallicity of the high$[\alpha / \mathrm{Fe}]$ stars, as stars with $|z| \geqslant 1 \mathrm{kpc}$ are slightly more metalpoor than stars close to the plane. This trend is identical to what is seen in $\mathrm{H} 15$; while all the high- $[\alpha / \mathrm{Fe}]$ stars formed at roughly the same time, the stars at the greatest distance from the mid-plane formed toward the low end of the radial distribution $(\sim 3 \mathrm{kpc})$ and the early end of this period ( $\sim 9 \mathrm{Gyr}$ ago) when relatively less metal enrichment had occurred.

High- $[\alpha / \mathrm{Fe}]$ stars are necessarily generated in regions of vigorous star formation. This environment is naturally produced in the innermost region of the exponential disk as inside-out growth progresses. While large gas-rich disks may generate such vigorous star formation across their full extent, given timescale limitations on gas mixing, the likelihood that the resulting the high- $[\alpha / \mathrm{Fe}]$ MDFs would be chemically identical is small. Thus, for the MW, it is more plausible that vigorous star formation occurred within a relatively small volume of gas that was in a chemically well-mixed rather than in a globally well-mixed ISM, and the stars that were generated from this gas were then dispersed throughout the MW via radial migration. 


\section{DISCUSSION AND CONCLUSIONS}

We have analyzed the MDFs from a simulation of a disk galaxy that experiences significant radial migration and compared them to recent results from the APOGEE survey. From this, we draw two main conclusions: (1) in both the simulation and APOGEE data, the skewness of the MDF shifts as a function of radius, most notably in the mid-plane of the disk. In the interior of the disk, the shape of the MDF is skewed toward low metallicities, and outside the solar cylinder, the shape of the MDF is skewed toward high metallicities. This shift in the skewness is a natural consequence of migration by spiral corotation and requires no fine-tuning to reproduce. (2) Regardless of the radial bin considered, the MDFs of the high$[\alpha / \mathrm{Fe}]$ stars are identical. This is because these stars all formed at roughly the same place at the same early time, and subsequently were spread throughout the disk. We suggest that comparing the peak metallicity of gas in a volume to the metallicity and kinematics of stars could be useful in identifying outward migrators if the metallicity of the stars are significantly higher than the peak metallicity of the gas.

We thank the anonymous referee and Eric Bell for constructive feedback. S.R.L. acknowledges support from the Michigan Society of Fellows. V.P.D. is supported by STFC Consolidated grant ST/M000877/1. D.L.N. was supported by a Dean B. McLaughlin Fellowship at the University of Michigan. J.A.H. and M.R.H. acknowledge support from NSF award AST-1109178. M.V. is supported by HST-AR13890, NASA ATP award NNX15AK79G, and NSF Award AST-1515001. Funding for SDSS-III has been provided by the Alfred P. Sloan Foundation, the Participating Institutions, the National Science Foundation, and the U.S. Department of Energy Office of Science. The SDSS-III website is http:// www.sdss3.org/. SDSS-III is managed by the Astrophysical Research Consortium for the Participating Institutions of the SDSS-III Collaboration.

\section{REFERENCES}

Alam, S., Albareti, F. D., Allende Prieto, C., et al. 2015, ApJS, 219, 12 Anders, F., Chiappini, C., Santiago, B. X., et al. 2014, A\&A, 564, A115 Azzalini, A., \& Capitanio, A. 2009, arXiv

Bovy, J., Nidever, D. L., Rix, H.-W., et al. 2014, ApJ, 790, 127

Daniel, K. J., \& Wyse, R. F. G. 2015, MNRAS, 447, 3576

Debattista, V. P., van den Bosch, F. C., Roškar, R., et al. 2015, MNRAS, 452, 4094

Doane, D. P., \& Seward, L. E. 2011, J. Stat. Educ., 19, 2, http://www.amstat. org/publications/jse/v19n2/doane.pdf

Eisenstein, D. J., Weinberg, D. H., Agol, E., et al. 2011, AJ, 142, 72

Fuhrmann, K. 2011, MNRAS, 414, 2893

Grand, R. J. J., Kawata, D., \& Cropper, M. 2015, MNRAS, 447, 4018

Hayden, M. R., Bovy, J., Holtzman, J. A., et al. 2014, AJ, 147, 116

Hayden, M. R., Holtzman, J. A., Bovy, J., et al. 2015, ApJ, 808, 132

Holmberg, J., Nordström, B., \& Andersen, J. 2009, A\&A, 501, 941

Holtzman, J. A., Shetrone, M., Johnson, J. A., et al. 2015, AJ, 150, 148

Liu, C., \& van de Ven, G. 2012, MNRAS, 425, 2144

Loebman, S. R., Roškar, R., Debattista, V. P., et al. 2011, ApJ, 737, 8

Minchev, I., \& Famaey, B. 2010, ApJ, 722, 112

Navarro, J. F., Frenk, C. S., \& White, S. D. M. 1997, ApJ, 490, 493

Nidever, D. L., Bovy, J., Bird, J. C., et al. 2014, ApJ, 796, 38

Raiteri, C. M., Villata, M., \& Navarro, J. F. 1996, A\&A, 315, 105

Ramírez, I., Allende Prieto, C., \& Lambert, D. L. 2013, ApJ, 764, 78

Roškar, R., Debattista, V. P., \& Loebman, S. R. 2013, MNRAS, 433, 976

Roškar, R., Debattista, V. P., Quinn, T. R., Stinson, G. S., \& Wadsley, J. 2008a, ApJL, 684, L79

Roškar, R., Debattista, V. P., Quinn, T. R., \& Wadsley, J. 2012, MNRAS, 426, 2089

Roškar, R., Debattista, V. P., Stinson, G. S., et al. 2008b, ApJL, 675, L65

Schönrich, R., \& Binney, J. 2009, MNRAS, 399, 1145

Sellwood, J. A., \& Binney, J. J. 2002, MNRAS, 336, 785

Smagorinsky, J. 1963, MWRv, 91, 99

Solway, M., Sellwood, J. A., \& Schönrich, R. 2012, MNRAS, 422, 1363

Steinmetz, M., Zwitter, T., Siebert, A., et al. 2006, AJ, 132, 1645

Stinson, G., Seth, A., Katz, N., et al. 2006, MNRAS, 373, 1074

Thielemann, F.-K., Nomoto, K., \& Yokoi, K. 1986, A\&A, 158, 17

Vera-Ciro, C., D’Onghia, E., Navarro, J., \& Abadi, M. 2014, ApJ, 794, 173

Wadsley, J. W., Stadel, J., \& Quinn, T. 2004, NewA, 9, 137

Wadsley, J. W., Veeravalli, G., \& Couchman, H. M. P. 2008, MNRAS, 387,427

Weidemann, V. 1987, A\&A, 188, 74

Yanny, B., Rockosi, C., Newberg, H. J., et al. 2009, AJ, 137, 4377

Yu, J., Sellwood, J. A., Pryor, C., Chen, L., \& Hou, J. 2012, ApJ, 754, 124 